\title{
674.
}

\section{NOTE ON THE CONSTRUCTION OF CARTESIANS.}

[From the Quarterly Journal of Pure and Applied Mathematics, vol. xv. (1878), p. 34.]

IF $\rho=a+b \cos \theta$, and $r=\frac{1}{2}\left\{\rho \pm \sqrt{ }\left(\rho^{2}-c^{2}\right)\right\}$, then obviously $r^{2}-r \rho+\frac{1}{4} c^{2}=0$, that is,

$$
r^{2}-r(a+b \cos \theta)+\frac{1}{4} c^{2}=0,
$$

which is the equation of a Cartesian. Here $\rho=a+b \cos \theta$ is the equation of a limaçon or nodal Cartesian, having the origin for the node; and for any given value of $\theta$, deducing from the radius vector of the limaçon the new radius vector $r$ by the above formula $r=\frac{1}{2}\left\{\rho \pm \sqrt{ }\left(\rho^{2}-c^{2}\right)\right\}$, we obtain a Cartesian, or by giving different values to $c$, a series of Cartesians having the origin for a common focus. The construction is a very convenient one. 